\title{
Feasibility of Manual Teach-and-Replay and Continuous Impedance Shaping for Robotic Locomotor Training Following Spinal Cord Injury
}

\author{
Jeremy L. Emken*, Susan J. Harkema, Janell A. Beres-Jones, Christie K. Ferreira, and \\ David J. Reinkensmeyer, Member, IEEE
}

\begin{abstract}
Robotic gait training is an emerging technique for retraining walking ability following spinal cord injury (SCI). A key challenge in this training is determining an appropriate stepping trajectory and level of assistance for each patient, since patients have a wide range of sizes and impairment levels. Here, we demonstrate how a lightweight yet powerful robot can record subject-specific, trainer-induced leg trajectories during manually assisted stepping, then immediately replay those trajectories. Replay of the subject-specific trajectories reduced the effort required by the trainer during manual assistance, yet still generated similar patterns of muscle activation for six subjects with a chronic SCI. We also demonstrate how the impedance of the robot can be adjusted on a step-by-step basis with an error-based, learning law. This impedance-shaping algorithm adapted the robot's impedance so that the robot assisted only in the regions of the step trajectory where the subject consistently exhibited errors. The result was that the subjects stepped with greater variability, while still maintaining a physiologic gait pattern. These results are further steps toward tailoring robotic gait training to the needs of individual patients.
\end{abstract}

Index Terms-Adaptive control, legged locomotion, motor systems, robots, spinal cord injury.

\section{INTRODUCTION}

$\mathbf{L}$ OCOMOTOR training using body weight support and a treadmill (BWST) is a well-established technique for gait recovery following neurological injury. First described in human clinical application by Barbeau et al. in 1987 [1],

Manuscript received September 27, 2006; revised April 7, 2007. This work was supported by ATP 00-00-4906 and the NIDRR RERC on Spinal Cord Injury under Grant H133E020732. The work of J. L. Emken was supported by an Achievement Reward for College Scientists Scholarship. Asterisk indicates corresponding author.

*J. L. Emken was with the Biomedical Engineering Department, University of California, Irvine, Irvine, CA 92797 USA. He is now with the Division of Biology, California Institute of Technology, MC 216-76, Pasadena, CA 91125 USA (e-mail: emken@ caltech.edu).

S. J. Harkema is with the Department of Neurological Surgery, Frazier Rehab Institute, University of Louisville, Louisville, KY 40202 USA (e-mail: susan. harkema@jhsmh.org).

J. A. Beres-Jones is with the Department of Neurology and Brain Research Institute, University of California, Los Angeles, Los Angeles, CA, 90095 USA (e-mail: jaberes@comcast.net).

C. K. Ferreira is with the Department of Neurological Surgery, University of Louisville, Frazier Rehab Institute, Louisville, KY 40202 (e-mail: ckferr01@gwise.louisville.edu).

D. J. Reinkensmeyer is with the Mechanical and Aerospace Engineering Department and the Biomedical Engineering Department, University of California, Irvine, Irvine, CA 92797 (e-mail: dreinken@ uci.edu).

Digital Object Identifier 10.1109/TBME.2007.910683 this technique has shown promise promoting gait restoration for both stroke [2], [3] and spinal cord injury (SCI) [4], [5] patients. This technique has also enabled researchers to study and understand mechanisms of spinally mediated walking [3], [6], [9]. BWST enables neurologically injured patients to safely practice repetitive stepping in a controlled clinical setting by providing body weight support (BWS) from an overhead harness and manual assistance to the pelvis and legs as a subject walks on a treadmill. The facilitated sensory input associated with the stepping presumably interacts with spinal interneurons to generate a locomotor pattern, as has been shown in animals [10]-[12]. Phasic loading and unloading of the limbs in concert with hip extension during terminal stance have been suggested as the major peripheral drives [3], [6], [9]. While extended practice with BWST is beneficial to patients, the technique is highly labor-intensive requiring strenuous and nonergonomic work from up to three trainers.

In order to assist the trainers in delivering manual assistance, several researchers and companies are developing robotic technology to automate manual assistance during locomotor training [13]-[18]. The first two devices to undergo clinical testing, that have been reported in the literature, are the Mechanized Gait Trainer (GTII) [15], [17] and the Lokomat [13], [14]. These devices were developed to reduce the trainer's workload and provide repeatable stepping kinematics of the legs. The GTII is a singly actuated mechanism that drives the subject's feet through a step-like trajectory using a doubled crank and rocker system. Vertical and horizontal movements of the pelvis are controlled in a phase dependent manner by ropes attached to the subject's harness. Gait trajectories are a fixed function of the crank and rocker geometry. In contrast, the Lokomat is a "driven gait orthosis" (DGO) (i.e., a robotic exoskeleton) that assists hip and knee flexion to again create step-like patterns. It consists of four rotary joints powered through four precision ball screws driven by dc motors. The orthosis is attached to the subject with a belt around the waist and cuffs around the thigh and shank. Movement of the hips is allowed only vertically. Ankle plantarflexion is constrained during swing phase with foot straps. Desired trajectories are taken from a database of healthy subjects who stepped over 3-cm-high obstacles while secured in a modified version of the DGO [13].

While current forms of robotic BWST during treadmill stepping can elicit significant improvements in ambulation ability and functional test scores with repetitive practice following stroke [15], [17] and SCI [8], [19], [20], they likely do not do 
so in an optimal fashion [21]-[23]. Both the Lokomat and to a lesser extent the GTII, constrain the degrees of freedom at the hip. Thus, these devices hinder pelvic movement, which is a normal biomechanical gait feature that may facilitate the neural control of walking. The crank-and-rocker mechanism of the GTII, while simple, prevents the abrupt transition in ground contact force between stance and swing that also may be important in facilitating flexor/extensor transitions in spinal locomotor circuits [3], [6], [9]. Further, the Lokomat has sizeable endpoint impedance, and the GTII constrains leg movement to a mechanically fixed trajectory. Thus, for both devices, the subject must step along a prerecorded or a mechanically fixed trajectory, rather than a trajectory customized specifically to their limb anthropometry and ability as is experienced with BWST. Further, because of the sizeable endpoint impedance, the individual has little flexibility to modulate their muscle activity and thus to directly and immediately experience the results in terms of altered movement kinematics and balance. As an example of the current systems' non-optimality, the Lokomat system produced significantly different [22] electromyography (EMG) patterns when attached compared to unassisted stepping for unimpaired subjects. Despite these possible shortcomings, robotic assistance with these devices eases the workload of trainers and can produce positive training results [14], [24].

The problem of customizing the level of robotic assistance to the individual stepping characteristics of a patient is being addressed with a newer version of the GTII concept. The new version uses force feedback to control foot platforms that can move along arbitrary stepping paths [25]. The same problem is also being addressed for the Lokomat by devising software algorithms that make the robot's assistance "patient-cooperative" [21], [26]. The first versions of the Lokomat used a stepping pattern recorded from a healthy subject, measured with the actuators of the device removed. Removing actuators for such "teach-and-replay" was impractical for regular laboratory use, so algorithms were developed for the Lokomat that allowed clinicians to adjust the step shape on a subject-specific basis. Specifically, a force-sensing algorithm is used to reduce the overall impedance of the robot, allowing more variability in the stepping trajectory. A second algorithm adjusts the reference trajectory and impedance of the robot for the patient, again based on the interaction force between the patient and robot. Finally, software has been developed for the Lokomat to provide visual biofeedback to the patient regarding their level of effort as gauged by the force sensing functions [26]. The approach taken here is different in that the device allows straightforward measurement and immediate replay of the pattern a trainer determines as optimal, incorporating the knowledge and expertise of the clinician into the training session. The most optimal training strategies for specific patients with different pathologies of gait are continuously under investigation. This robotic device was designed with the intention to provide the ability to vary the control algorithm for each patient and allow variability as the patient improves, even allowing the ability to vary the assistance within a session. A future question that requires additional research is whether "healthy stepping" from a generic individual, or manually assisted "pathological stepping" from the specific individual to be trained, will produce better clinical results.
We are also attempting to customize the level of robotic assistance to the individual stepping characteristics of a patient by designing robotic gait training systems that allow naturalistic kinematic and loading patterns [18], [27]-[29]. This paper reports the first clinical testing of a novel gait robot, the "AmbulationAssisting Robotic Tool for Human Rehabilitation" (ARTHuR), with spinal cord injured subjects. ARTHuR is a highly backdrivable yet powerful robot capable of measuring and manipulating human stepping [18]. We tested the feasibility of generating participant-specific stepping patterns by using this ambulation-assisting robot in a "teach-and-replay" mode. Specifically, we used the device to record the stepping kinematics of the leg, dictated by a human trainer during manually assisted stepping with the robot passively attached. The recorded kinematics were then replayed to allow for reduced effort from the human trainer. We compared the leg muscle activity patterns elicited during trainer-assisted and robot-assisted stepping, as an assessment of how effective the device was in eliciting step activity.

We also tested an adaptive algorithm for shaping the impedance of the robot controller as a function of tracking performance in real time. We show that this algorithm reliably finds workspace-dependent patterns of impedance that allow greater step-to-step variability without compromising the overall kinematic pattern of stepping.

\section{METHODS}

\section{A. Subject Population}

Six individuals with a chronic SCI participated in the study. An experienced assessor evaluated the level and extent of SCI according to the American Spinal Injury Association (ASIA) impairment scale. This scale categorizes individuals by their sensorimotor function below the level of the lesion and designates a sensory and motor score [30]. All subjects had previously received some amount of BWST. None of the subjects were taking antispasticity medication at the time of the experiment. Injury level, years post injury, and ASIA impairment levels for each subject are summarized in Table I. The Institutional Review Boards of the Universities of California at Irvine and Los Angeles approved the experimental protocol and each subject signed an informed consent form prior to participating in the study. A limited population and only subjects with ASIA scores in the range of B-D were chosen, as this was a proof of concept test for the device and not a determination of clinical efficacy.

\section{B. Experimental Apparatus and Control: Teach-and-Replay}

We used a backdrivable two degrees-of-freedom planar robot ("ARTHuR" [18]) to measure and assist in the stepping of the right leg. ARTHuR is currently a unilateral device and thus for the purposes of the present feasibility study, only attachment to the right leg was studied. This robot uses a two-coil linear motor and a pair of lightweight linkages to drive the robot's apex, which is attached to the subject through a revolute joint and running shoe modified to include an embedded footplate (Fig. 1). The real-time controller for the system uses two computers, a host machine running Windows and a target machine running 
TABLE I

SUBJECT CHARACTERISTICS

\begin{tabular}{|c|c|c|c|c|c|c|}
\hline Subject & Sex & Age & $\begin{array}{c}\text { Lesion } \\
\text { Level }\end{array}$ & $\begin{array}{c}\text { Years } \\
\text { Post } \\
\text { Injury }\end{array}$ & ASIA & $\begin{array}{c}\text { BWS } \\
\mathbf{( \% )}\end{array}$ \\
\hline SCI-C8 & $\mathrm{M}$ & 23 & C5-6 & 4 & C & 62 \\
\hline SCI-B4 & $\mathrm{M}$ & 31 & T4-6 & 11 & B & 70 \\
\hline SCI-D8 & M & 32 & C5-6 & 15 & D & 40 \\
\hline SCI-D9 & F & 54 & C6 & 10 & D & 29 \\
\hline SCI-D10 & F & 51 & C5 & 7 & D & 21 \\
\hline SCI-D7 & M & 61 & C6-7 & 6 & D & 25 \\
\hline
\end{tabular}

$\mathrm{C}=$ Cervical, $\mathrm{T}=$ Thoracic, BWS $=$ Percent of Body Weight Support. ASIA B: Sensory but not motor function is preserved below the neurological level.

ASIA C: Motor function is preserved below the level of injury, and more than half of key muscles below the level of injury have a muscle grade less than three.

Asia D: Motor function is preserved below the neurological level, and at least half of key muscles below the neurological level have a muscle grade of three or more.

a Simulink program on a real time kernel called xPC. The Simulink control program is created and uploaded to the target machine, which connects to the robot through a D/A board (Measurement Computing, Norton, MA), an encoder board (Addi-Data, Ottersweier, Germany), and a custom I/O box containing the motor drivers, encoders, safety, and watchdog circuitry. Data is collected on the target computer in real-time and then transferred to the host machine later so that it can be processed using custom Matlab scripts. The device allows a full range of motion in the parasagittal plane during walking and includes a passive joint that allows approximately $10^{\circ}$ of hip adduction/abduction from the anatomical position. The shoe attachment constrains medial and lateral rotation of the hip and shank. To accommodate a shared workspace with the leg trainer, curved linkages were designed to allow the trainer to move naturally while assisting the movement of the knee and ankle (Fig. 1). To help prevent foot drop during swing, the robot's apex was positioned approximately one centimeter in front of the ankle joint center of rotation to impart a small dorsiflexion torque allowing for toe clearance during early swing phase. The apex's Cartesian position was calculated from the position of each coil measured at $1 \mathrm{kHz}$ by the coil-mounted, linear encoders each with a resolution of $20 \mu \mathrm{m}$. Coil velocities were calculated using the transfer function $=\mathrm{s} / 0.01 \mathrm{~s}+1$ on the coil positions and then were translated into the apex's coordinate frame for endpoint velocity. This real time differentiation did not induce significant noise into the velocity signals. A rotary encoder in the revolute joint measured angular rotation of the shoe around the apex, a measurement which was used along with shoe dimension and apex state to calculate toe position and velocity.

An important capability of this robot is its ability to accurately record the trajectories induced during manually assisted stepping by a leg trainer and replay a processed, mean trajectory using proportional-derivative (PD) control. We call this function "teach-and-replay". To allow for the teach-and-replay function, we first attached the robot to the subject in a "passive mode." During the passive mode, the robot did not assist stepping, and the effects of gravity and friction were actively

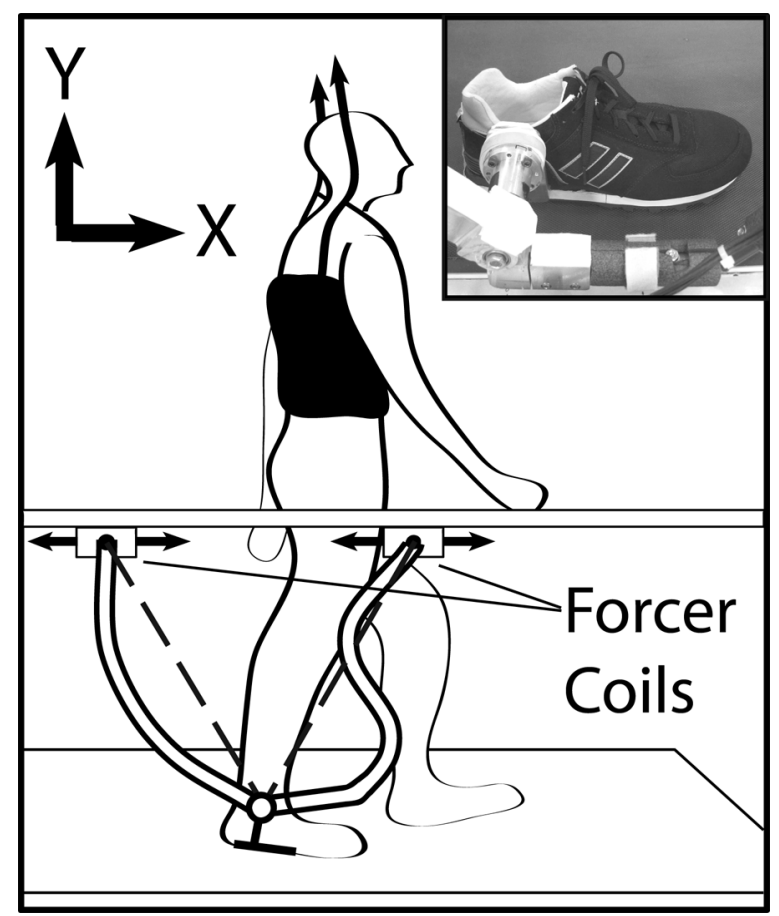

Fig. 1 Experimental setup and custom shoe attachment (inset). The robot design utilizes a linear motor with two forcer coils and a lightweight V-shaped linkage to drive the device's apex. The original V-shaped design of the robot linkages is shown for reference in dashed lines. New curved linkages allow robot and trainer to share the same workspace while they manipulated the leg. The upper curve of the forward linkage allow space for the trainer's upper arm during assistance at the knee, while the lower curve created an opening for the trainer's lower hand to assist at the ankle. Inset: The subjects wear a custom shoe with an embedded footplate that is attached to the robot's apex through a revolute joint.

cancelled within the software. We have shown previously that the robot only slightly alters the stepping trajectory of nondisabled subjects in "passive mode" [18]. The added inertia contributes to a slightly higher stepping trajectory compared to when the robot is not attached. The robot then recorded the position and velocity of the apex at $1 \mathrm{kHz}$ while the subject was manually stepped for a series of 30 steps. Following data segmentation into individual steps based on a zero crossing of the $x$ velocity signal, steps with durations exceeding $+/-8 \%$ of the original mean stepping duration were discarded. The steps were then normalized via interpolation using a cubic spline to the mean step duration. The state variables $(x, y, \dot{x}, \dot{y})$ were averaged across steps and a mean centroid in position space was calculated for each step and across steps. Steps whose centroid fell more than $1.25 \sigma$ from the overall mean centroid were then also discarded. The remaining steps were averaged, and interpolated to initiate the step during mid-stance. The resultant desired trajectory $\left(x_{d}, y_{d}, \dot{x}_{d}, \dot{y}_{d}\right)$ was used during active assist mode to drive the apex through the subject-specific trajectory with a PD controller running at $1 \mathrm{kHz}$ :

$$
\begin{aligned}
& F_{x}(t)=-k_{x}\left(x(t)-x_{d}(t)\right)-b_{x}\left(\dot{x}(t)-\dot{x}_{d}(t)\right) \\
& F_{y}(t)=-k_{y}\left(y(t)-y_{d}(t)\right)-b_{y}\left(\dot{y}(t)-\dot{y}_{d}(t)\right)
\end{aligned}
$$

where $F_{x}$ and $F_{y}$ are the robot forces, $k_{x}$ and $k_{y}$ are the position gains, $b_{x}$ and $b_{y}$ are the velocity gains, and $t$ is time. 
TABLE II

EXPERIMENTAL PROTOCOL

\begin{tabular}{|c|c|c|c|c|c|c|}
\hline \multirow[t]{2}{*}{ Experiment } & \multirow{2}{*}{$\begin{array}{l}\text { Full Manual } \\
\text { Assistance* } \\
\quad \text { (steps) }\end{array}$} & \multirow{2}{*}{$\begin{array}{c}\text { Combined } \\
\text { Manual and } \\
\text { Robot Assistance } \\
\text { (steps) }\end{array}$} & \multicolumn{4}{|c|}{$\begin{array}{c}\text { Right Leg Trainer } \\
\text { Assisting at Hand Placement during } \\
\text { combined assistance steps }\end{array}$} \\
\hline & & & Patella & Popliteal & TA & Achilles \\
\hline Warm up & $100-200$ & - & $\mathrm{X}$ & $\mathrm{X}$ & $\mathrm{X}$ & $\mathrm{X}$ \\
\hline A - Baseline & 30 & - & $\mathrm{X}$ & $\mathrm{X}$ & $\mathrm{X}$ & $\mathrm{X}$ \\
\hline B - Teach / Record & 30 & - & $X$ & $X$ & $\mathrm{X}$ & $\mathrm{X}$ \\
\hline C - Full Assistance ${ }^{\dagger}$ & 20 & 20 & $\mathrm{X}$ & $X$ & $\mathrm{X}$ & $\mathrm{X}$ \\
\hline D - No Ankle ${ }^{\dagger}$ & 20 & 20 & $\mathrm{X}$ & $\mathrm{X}$ & & \\
\hline E - Patella Only ${ }^{\dagger}$ & 20 & 20 & $\mathrm{X}$ & & & \\
\hline F - Patella Only - Adapt & 5 & $60^{\wedge}$ & $\mathrm{X}$ & & & \\
\hline G - Patella Only -Adapt & 5 & $60^{\wedge}$ & $\mathrm{X}$ & & & \\
\hline
\end{tabular}

We also developed two active assist modes for the device: static and adaptive. In static mode, the PD gains were held constant at: $k_{x}=70 \mathrm{~N} / \mathrm{cm} ; k_{y}=105 \mathrm{~N} / \mathrm{cm} ;$ and $b_{x}=b_{y}=3.5$ $\mathrm{N}-\mathrm{s} / \mathrm{cm}$. As a safety precaution, we chose the stiffness to be higher in the $y$ (vertical) direction to ensure toe clearance during swing. In adaptive mode, we shaped the gains of the PD controller as a function of stepping error, an approach based on our adaptive learning research with nondisabled subjects [28], [31]. Briefly, we have studied previously how healthy nondisabled subjects adapt to perpendicular viscous force fields applied to the leg with ARTHuR. We found that the human motor system can be modeled by implementing an error based control law with a forgetting factor $f$. The presence of the forgetting factor indicates that the human motor system continuously attempts to accomplish a desired movement with decreased effort, provided that the error was small on the previous movement attempt. This type of controller can be shown to minimize a cost function containing error and effort terms [32].

We desired to design a controller for the robotic step trainer that reduced the amount of assistance provided by the robot if stepping errors were small. In simulations, we had previously explored the use of a control law like the one we identified for the human motor system to shape the feedback gains of an assisting robot [33], [34]. We used a similar approach in our current design, shaping the stiffness and damping of the PD controller uniquely for each workspace location, based on subject position and velocity tracking errors measured in real time

$$
\begin{aligned}
& k_{x}(n+1, t)=f_{k} k_{x}(n, t)+g_{k}\left|\left(x(t)-x_{d}(t)\right)\right| \\
& k_{y}(n+1, t)=f_{k} k_{y}(n, t)+g_{k}\left|\left(y(t)-y_{d}(t)\right)\right| \\
& b_{x}(n+1, t)=f_{b} b_{x}(n, t)+g_{b}\left|\left(\dot{x}(t)-\dot{x}_{d}(t)\right)\right| \\
& b_{y}(n+1, t)=f_{b} b_{y}(n, t)+g_{b}\left|\left(\dot{y}(t)-\dot{y}_{d}(t)\right)\right|
\end{aligned}
$$

where $f$ is a scalar forgetting factor, $\left(f_{k}=f_{b}=0.90\right), g$ is a learning gain, $\left(g_{k}=18 \mathrm{~N} / \mathrm{cm}, g_{b}=0.18 \mathrm{~N}-\mathrm{s} / \mathrm{cm}\right), \mathrm{n}$ is step number, $x-x_{d}$, for example, is the position tracking error in the $x$ direction, and $t$ is time. The values of $f$ and $g$ were chosen such that the adaptive robot-human system would reach steady state after 30 step cycles with an approximate mean tracking error of $2 \mathrm{~cm}$. Shaping of the PD gains occurred as a function of the temporal index of the stepping profile $(t)$ and was updated per step $(n)$ based on the position error at the index $(t)$ of the previous step (2). In all actively controlled steps, the desired trajectory was synchronized to the actual trajectory at the middle of stance to account for alterations in step duration or step length.

Redundant safety features were incorporated into the design and operation of the robot. These features included software position and velocity limits, an independent watchdog timer to monitor the drives and computer communication, an operator-held, emergency stop button, and motor drive and encoder checks that generated a robot shutdown if detected [18]. In addition, the software saturated the desired, open loop forces to 44 and $89 \mathrm{~N}$ in the $x$ and $y$ directions, respectively, which are force levels that the trainer could overcome if necessary. The shoe rotation sensor at the robot's apex allowed for the detection of subject toe stub following foot drop at the beginning of stance, as well as excessive plantar flexion of the ankle $\left(>105^{\circ}\right.$ - anatomical position of the ankle is $0^{\circ}$ ). During replay, window limits of $5 \mathrm{~cm}$ and $25 \mathrm{~cm} / \mathrm{s}$ were set on the position and velocity tracking errors, respectively. The control software shut down the robot if it detected any fault condition. This shutdown returned the robot to its passive, backdrivable state without friction and gravity cancellation.

\section{Experimental Design}

All subjects wore a harness (Robertson Mountaineering) and were suspended by an overhead, force-controlled, pneumatic BWS system lift (Robomedica, Inc.). Level of BWS (Table I) was adjusted for each subject by an experienced physical trainer based on the quality of stepping and to maintain subject safety. Once established, the level of BWS for each subject was held constant throughout the experiments.

During all experiments, experienced trainers provided manual assistance at the pelvis, knees, and ankles as necessary to promote good stepping. Hand placement distal to the patella assisted in knee extension during the stance phase, and at the popliteal crease for hip and knee flexion during swing. Hand placement proximal to the ankle assisted proper foot placement and foot clearance at liftoff. A trainer positioned behind the subject aided in pelvis stabilization and weight shifting during stepping. The pelvis and left leg trainers attempted to provide consistent patterns of assistance across experimental conditions, while the right leg trainer altered the pattern of manual assistance according to a series of experimental conditions 
described below (Table II). Data were recorded during stepping at a fixed treadmill speed of $0.89 \mathrm{~m} / \mathrm{s}$. SCI-D10, a shorter subject, stepped at a fixed treadmill speed of $0.80 \mathrm{~m} / \mathrm{s}$.

All subjects completed seven experiments in a single 2-h session. The experiments were designed to test the effect of the robot attachment on stepping activity, the effect of alterations in manual assistance with robot assistance on stepping activity, and the ability of the impedance shaping algorithm described above to adapt to the individual subject's stepping needs (Table II). All subjects first stepped for 100-200 steps in a warm up period without the robot attached. Following the warm up period, we measured EMG patterns associated with manually assisted stepping without the robot attached to establish a baseline for further comparison (Experiment A). Data were recorded for 30 steps. Experiment B was used as a robot training session that allowed the trainer to manually teach the robot the desired step trajectory for each subject. This entailed attaching the robot's apex to the custom shoe and stepping the subject with manual assistance for 30 steps, with the robot in passive mode. The recorded steps from experiment B were averaged and filtered to generate a mean stepping trajectory. Experiments $\mathrm{C}-\mathrm{G}$ used this mean stepping trajectory as the desired trajectory for the PD controller (1) to replay the recorded steps. For each of these experiments, the trainers manually stepped the subjects for a fixed number of steps after which the robot switched from passive to active mode and began actively assisting using the PD controller. The right leg trainer then altered the placement of their hands in accordance with the protocol shown in Table II within one to two steps. In Experiment $\mathrm{C}$, the trainer continued to assist as needed at both the ankle and knee during stance and swing. In Experiment D, the trainer assisted only at the knee (no ankle assistance) but both in extension and flexion. In Experiment E, the trainer assisted only knee extension during stance. In all cases, the trainer assisted only when needed. The order of experiments $\mathrm{C}, \mathrm{D}$, and $\mathrm{E}$ was randomized across subjects. The gains of the PD controller were held constant (static mode) for experiment C-E.

In the final two experiments ( $F$ and $G$ ), the feedback gains of the PD controller were shaped according to (2). Assistance was provided with the controller gains fixed (static mode) for ten steps after which the mode was switched to adaptive and the algorithm shaped the gains for 50 steps. The trainer also switched from full manual assistance to assisting only at the patellar tendon (knee extension) after the first ten steps. So, while the gains were adapting, the trainer only helped when needed to prevent knee flexion during the stance phase of the gait cycle. This experiment was replicated to test for the repeatability of convergence of the PD gains. Five of the six subjects successfully completed these two experiments, stepping 60 consecutive steps in each experiment. Subject SCI-D10 developed excessive hip adduction when the trainer stopped assisting at the ankle after ten steps, and was not included in the analysis, as movements out of the parasagittal plane caused errors that repeatedly triggered safety faults after 20-30 steps in the adaptive field. These experiments were designed as a proof of concept of the teach-and-replay and adaptive impedance shaping algorithms and not to test the therapeutic efficacy of the device.

\section{Data Acquisition}

For all subjects, we measured EMG activity, level of body weight support, and the kinematic state (Cartesian position and velocity) of the apex and toe of the right foot when the robot was attached. The force applied to the subject by the robot was estimated from the motor currents, a technique that accurately estimates these forces since the robot is lightweight and has low friction [18]. Kinematic and force data from the robot were collected at $1 \mathrm{kHz}$ using a custom-written Simulink-xPC software control and acquisition program (The Mathworks, Inc., Natick, MA). EMG, footswitch, and BWS data were collected on a separate computer at $1 \mathrm{kHz}$ using a custom Labview (National Instruments, Austin, TX) software acquisition program. EMG data were sampled and ac-coupled into a differential amplifier (Konisberg Instruments, Pasadena, CA). We measured EMG activity bilaterally from the soleus (SL), medial gastrocnemius (MG), tibialis anterior (TA), medial hamstrings $(\mathrm{MH})$, vastus lateralis (VL), and rectus femoris (RF) using bipolar surface electrodes. The electrode placements have been described previously [6]. We verified the efficacy of electrode placement in order to avoid cross-talk contamination among muscles by eliciting and recording EMG activity in an individual muscle while recording inactivity in the other sampled muscles. BWS was recorded from a load cell in series with the cable attached to the subject's harness. Joint angles were not measured.

\section{E. Data Analysis}

Data synchronization and reduction were performed with the use of custom written software in Matlab and Labview. The EMG data were processed by high pass filtering at $32 \mathrm{~Hz}$ and rectifying. The data were divided into step cycles dependent on heel strike as measured by the foot contact switches and spatially checked with apex and toe position data from the robot when available (Experiment $\mathrm{C}-\mathrm{G}$ ).

Waveforms of EMG activity from the last ten steps of each condition (Experiments A-E) and all the steps of Experiment $F$ and $\mathrm{G}$, were processed to investigate relationships across conditions. First, data from individual steps were normalized to the mean cycle duration, as measured from heel strike to heel strike of the same foot, for each condition and then expressed as percent of total step cycle duration. The individual signals were then smoothed with a cubic spline. Finally, a mean representative signal with standard deviations was calculated from the average of the normalized and smoothed signals for each condition. This allowed for general comparisons across subjects, muscles, and experimental conditions.

Kinematic data were used to calculate step height, step length, and tracking error with respect to the desired stepping trajectory as a function of time for each step. The error and recorded PD gains were used to calculate the amount of open-loop force (1) applied to the subject, taking into account the force safety limits imposed by the software. Resultant forces were calculated from the $x$ and $y$ force components.

Performed separately from above, EMG data for the right and left legs were again segmented into step cycles based on heel strike for each respective leg. For each step cycle, a burst analysis was performed [6], [35] to quantify activity for each muscle. 
Comparisons across conditions were made examining the mean burst amplitude, which was defined as the integral of the burst activity/burst duration.

\section{F. Statistical Analysis}

After the average EMG profile for each muscle was calculated for all conditions, a 95\% confidence interval was calculated around the mean EMG activity for each muscle and condition across subjects. Analysis of similar EMG activity was quantified by calculating the percent overlap of the $95 \%$ confidence intervals in the step cycle for each muscle across conditions and then taking the mean, standard deviation, and magnitude with respect to peak amplitude of each percentage overlap across subjects.

Five comparisons were made with a one-way analysis of variance (ANOVA) on the mean burst amplitude. Each comparison was performed for each muscle of each subject. The first comparison analyzed the effect of passive robot attachment under full manual assistance with respect to full manual assistance as needed. The second, third, and fourth comparisons analyzed the effect of different assistance styles with robotic assistance provided at the shoe with respect to full manual assistance with the robot attached yet passive. The comparisons were: 2) full assistance as needed from the trainer, with the robot assisting; 3 ) assistance as needed only at the knee from the trainer, with the robot assisting; and 4) assistance as needed only at the patella from the trainer, with the robot assisting. The final comparison analyzed the effect of a compliant controller with respect to a stiff controller under robotic assistance coupled with assistance as needed only at the patellar tendon. For the nonattached-attached comparison, three comparisons were made, since there were three sets of steps with the robot attached but passive (beginnings of Experiments C, D, and E); likewise, two comparisons were made for the stiff compliant comparisons in Experiments $\mathrm{F}$ and $\mathrm{G}$. The individual comparisons were made using ANOVA and the overall comparison was only considered significantly different if all of the individual comparisons were significantly different. When significant, the mean percent difference between the starting condition and the mean of the repeated analyses was calculated.

\section{RESULTS}

\section{A. Kinematics and Muscle Activity for Manually Assisted Stepping}

Following a warm-up period during which the trainers manually assisted stepping without the robot attached, the robot's apex was attached to the subject's shoe and the trainers again manually assisted in stepping with the passive robot recording the foot trajectories. The trainers' subjective impression was that the robot was lightweight and did not interfere with their ability to manipulate the subject's leg. As expected, the mean stepping trajectory and position-velocity state space figures for each subject were unique, as a result of the interplay of the subject's own anthropometry, the subject's effort, and the trainer's assistance (Fig. 2). The EMG pattern differed among individuals with SCI during stepping with manual assistance (Fig. 3).
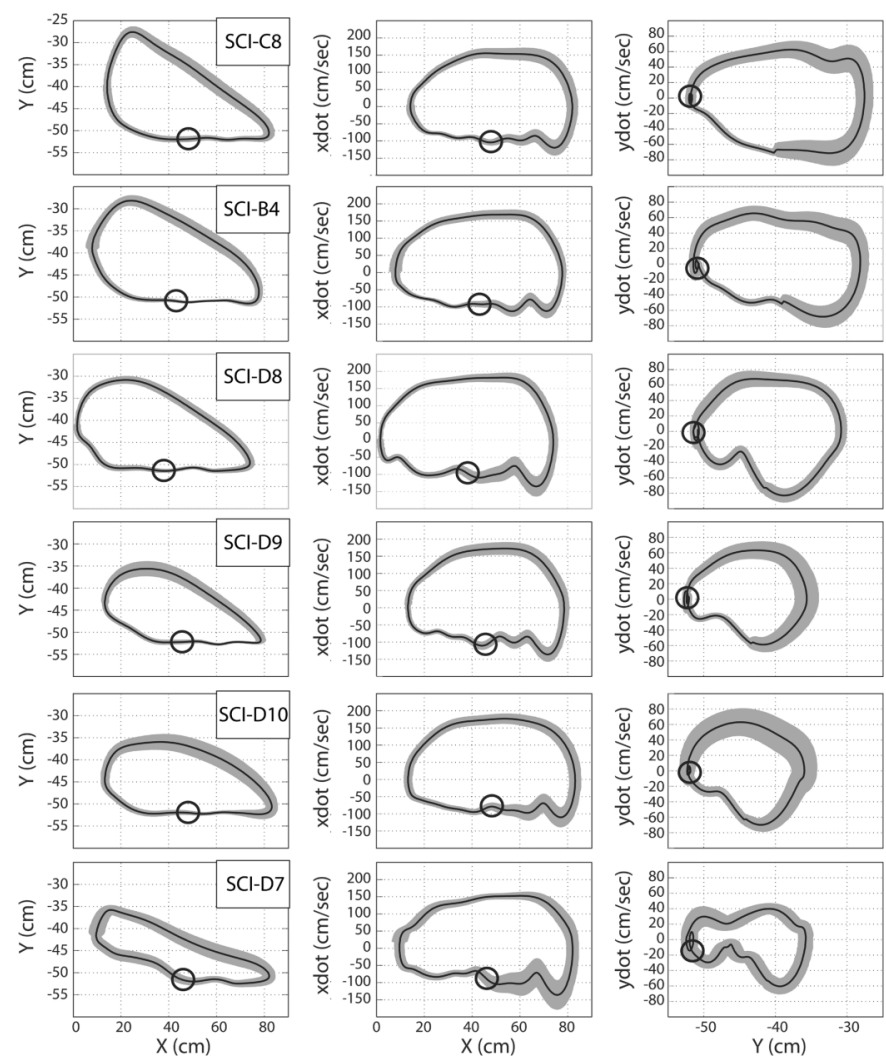

Fig. 2 Mean position and state trajectories recorded by the robot while the trainer manually stepped the six SCI subjects with the robot attached yet passive. Left: Position space plots are read clockwise and represent the stepping trajectory of the ankle as viewed from the right (subject facing to the right). Middle: $X$ Position $-X$ Velocity Space plots are also read clockwise. Right: $V$ Position $-Y$ Velocity space plots are read counter clockwise. The shaded areas around each mean trajectory represent the range of normal stepping fluctuations during manually assisted stepping (+/- three standard deviations). For reference, the circle marks middle of stance in all plots and the origin of the robot coincides with the position of the rear pivot upon initialization.
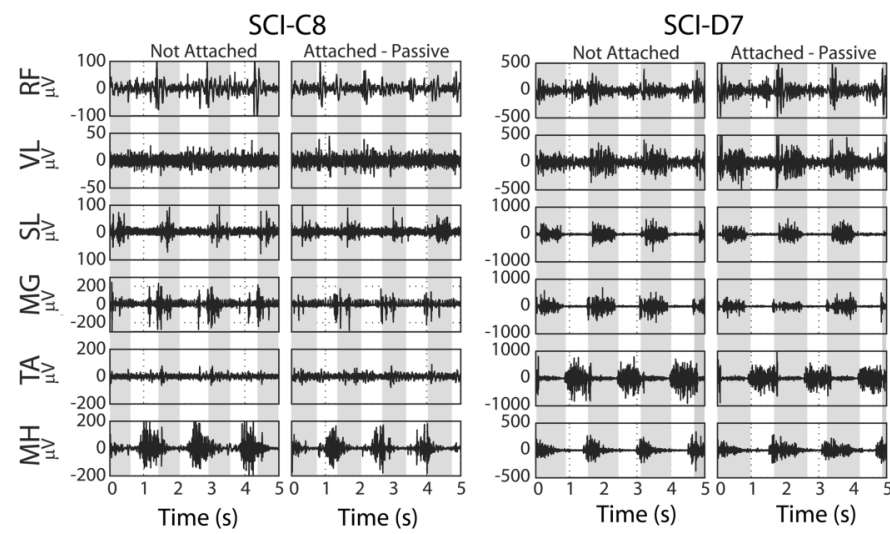

Fig. 3 Raw EMG for six muscles of the right leg for two representative SCI subjects for three consecutive steps. For each subject, the left panel shows EMG activity during stepping with manual assistance when the robot was not attached, and the right panel when the robot was attached and passive. In both cases, the trainers were providing assistance as needed to maintain the stepping pattern by the subject. Shaded areas represent stance phase. Abbreviations: soleus (SL), medial gastrocnemius (MG), tibialis anterior (TA), medial hamstrings $(\mathrm{MH})$, vastus lateralis (VL), and rectus femoris $(\mathrm{RF})$.

Attachment of the passive robot to the subject's leg generally had a small effect on the shape, magnitude, and timing 

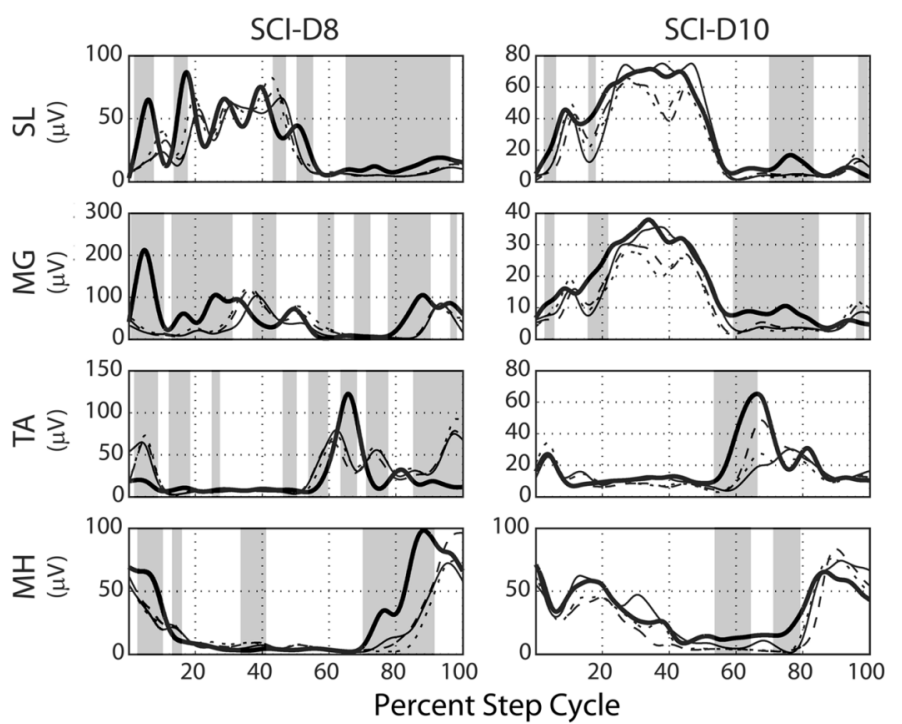

Fig. 4 Effect of passive robot attachment on patterns of muscle activation for two representative subjects. Thick solid line is without robot attached; thin solid, dotted, and dashed lines are with robot attached in passive mode in experiment C-E, respectively. Shaded areas correspond to phases of the step cycle in which there was consistently no overlap of the $95 \%$ confidence intervals comparing nonattached to attached yet passive conditions. Abbreviations: soleus (SL), medial gastrocnemius (MG), tibialis anterior (TA), medial hamstrings (MH).

of the mean EMG activity when examined visually, yet was numerically significantly different in parts as compared with the nonattached condition for (Figs. 3 and 4, Table III). The areas of the step cycle that consistently showed no overlap of 95\% confidence intervals across the three repetitions of the attached-yet-passive condition (i.e., the first parts of Exp C, D, and E) are represented in Fig. 4 by shaded regions for two representative subjects. The percentage of the step cycle for which the $95 \%$ confidence intervals did not overlap was $49+/-16$ $\%$. During these periods, the average absolute change in EMG amplitude from the nonattached to attached conditions was $23+$ / $-15 \%$, referenced to the peak nonattached EMG magnitude for the step cycle. On average, 53\% of the nonoverlap regions in EMG pattern occurred during stance phase when the foot was on the treadmill.

Analysis of the differences in the mean EMG burst amplitudes across the nonattached and attached conditions suggested that passive robot attachment only significantly affected two subjects ( $p<0.05$, Table III, SCI-D8 and SCI-D10, Fig. 4). The percent difference of the mean burst amplitudes was calculated with respect to the nonattached condition. As seen in Fig. 4, there were significant differences in the EMG waveforms but the general shape and amplitude were ostensibly similar. This analysis was confounded by the fact that over a quarter of the comparisons were unable to be calculated due to absence of activity or noise preventing measurement of individual bursts. In such cases, the background noise level was around 25-50 $\mu \mathrm{v}$.

\section{B. Comparison of Robot-Assisted and Manually Assisted Stepping}

1) Position Tracking Error: For the next series of experiments (Experiments C-E, Table II), the robot actively assisted in the subject's stepping, with the right leg trainer varying the
TABLE III

SUMMARY OF THE EFFECT OF EXPERIMENTAL CONDITION ON BURST AMPLITUDE

\begin{tabular}{|c|c|c|c|c|c|c|}
\hline \multicolumn{7}{|c|}{ Comparison 1: Effect of robotic attachment } \\
\hline & \multicolumn{6}{|c|}{ Muscle } \\
\hline Subject & SL & MG & TA & MH & $\mathrm{VL}$ & RF \\
\hline SCI-C8 & - & - & & - & & \\
\hline SCI-B4 & - & & - & & & \\
\hline SCI-D8 & - & $38 \% \downarrow$ & $152 \% \uparrow$ & $29 \% \downarrow$ & - & $71 \% \uparrow$ \\
\hline SCI-D9 & - & - & & - & & \\
\hline SCI-D10 & - & - & $42 \% \downarrow$ & - & $34 \% \downarrow$ & - \\
\hline SCI-D7 & - & - & - & - & - & - \\
\hline \multicolumn{7}{|c|}{ Comparison 2: Effect of robotic assistance and full manual assistance } \\
\hline & \multicolumn{6}{|c|}{ Muscle } \\
\hline Subject & $\mathrm{SL}$ & MG & TA & MH & VL & RF \\
\hline SCI-C8 & - & - & & $26 \% \downarrow$ & & \\
\hline SCI-B4 & $16 \% \downarrow$ & & - & & & \\
\hline SCI-D8 & - & - & $16 \% \downarrow$ & - & - & - \\
\hline SCI-D9 & - & - & & $9.1 \% \downarrow$ & & \\
\hline SCI-D10 & - & - & $60 \% \uparrow$ & - & - & $26 \% \uparrow$ \\
\hline SCI-D7 & $18 \% \uparrow$ & $29 \% \uparrow$ & $10 \% \uparrow$ & $24 \% \uparrow$ & - & - \\
\hline \multicolumn{7}{|c|}{$\begin{array}{c}\text { Comparison 3: Effect of robotic assistance and manual assistance only at the } \\
\text { knee }\end{array}$} \\
\hline & \multicolumn{6}{|c|}{ Muscle } \\
\hline Subject & SL & MG & TA & MH & VL & RF \\
\hline SCI-C8 & - & - & & $25 \% \downarrow$ & & \\
\hline SCI-B4 & $15 \% \downarrow$ & & - & & & \\
\hline SCI-D8 & $13 \% \downarrow$ & $14 \% \downarrow$ & $13 \% \uparrow$ & - & - & $18 \% \uparrow$ \\
\hline SCI-D9 & $22 \% \uparrow$ & - & & - & & - \\
\hline SCI-D10 & - & - & - & - & - & $8.8 \% \downarrow$ \\
\hline SCI-D7 & $12 \% \uparrow$ & - & - & $16 \% \downarrow$ & - & \\
\hline \multicolumn{7}{|c|}{$\begin{array}{c}\text { Comparison 4: Effect of robotic assistance and manual assistance only at the } \\
\text { patellar tendon }\end{array}$} \\
\hline & \multicolumn{6}{|c|}{ Muscle } \\
\hline Subject & SL & MG & TA & MH & VL & RF \\
\hline SCI-C8 & $17 \% \downarrow$ & - & & $25 \% \downarrow$ & & \\
\hline SCI-B4 & $21 \% \downarrow$ & & & & - & \\
\hline SCI-D8 & $14 \% \downarrow$ & $13 \% \downarrow$ & $14 \% \downarrow$ & $17 \% \downarrow$ & $35 \% \downarrow$ & $16 \% \downarrow$ \\
\hline SCI-D9 & $12 \% \downarrow$ & $25 \% \downarrow$ & & $17 \% \uparrow$ & & \\
\hline SCI-D10 & - & - & $27 \% \uparrow$ & - & $26 \% \downarrow$ & $15 \% \downarrow$ \\
\hline SCI-D7 & - & - & - & - & - & \\
\hline \multicolumn{7}{|c|}{$\begin{array}{l}\text { Shaded Grey }- \text { no detectable burst either due to absence of activity or high noise }(25-50 \mathrm{~m} \\
-: \text { No significant difference when compared with one-way ANOVA } \\
\% \text { : Significant difference ( } \mathrm{p}<0.05 \text { when compared with one-way ANOVA). Comparisons are made with } \\
\text { respect to full manual assistance in the non-attached (comparison } 1 \text { ) and attached yet passive conditions } \\
\text { (comparisons 2-4). }\end{array}$} \\
\hline
\end{tabular}

style of their manual assistance. In all cases, the trainers assisted only as needed altering their assistance to the needs of each individual. The kinematic pattern of stepping, as measured by the resultant position tracking error of the robot's apex, varied along the entire trajectory by an average across subjects of $0.81 \pm 0.49$ $\mathrm{cm}, 1.03 \pm 0.75 \mathrm{~cm}$, and $1.18 \pm 0.97 \mathrm{~cm}$ for experiments $\mathrm{C}, \mathrm{D}$, and $\mathrm{E}$, respectively. This indicates that each of the various combinations of trainer and robot assistance allowed the subject's leg to successfully move along the desired stepping trajectory. In all conditions and for all subjects the mean position tracking errors fell within one standard deviation of the respective desired trajectory. This result includes Experiment E, in which the trainer was only required to assist in knee extension during stance. In this case, the robot was solely responsible for assisting the leg through the swing motion.

2) Muscle Activity: Neither the mode of the robot nor the style of hand placement in which the right leg trainer assisted had major effects on the patterns of muscle activation (Fig. 5). Fig. 5 shows the regions of the step cycle in which the $95 \%$ confidence intervals of the EMG for the passive and active conditions did not overlap for two subjects. The mean percent values of consistently no overlap were $21 \pm 12 \%$ for the condition of full trainer assistance, $20 \pm 10 \%$ for the condition of no ankle 

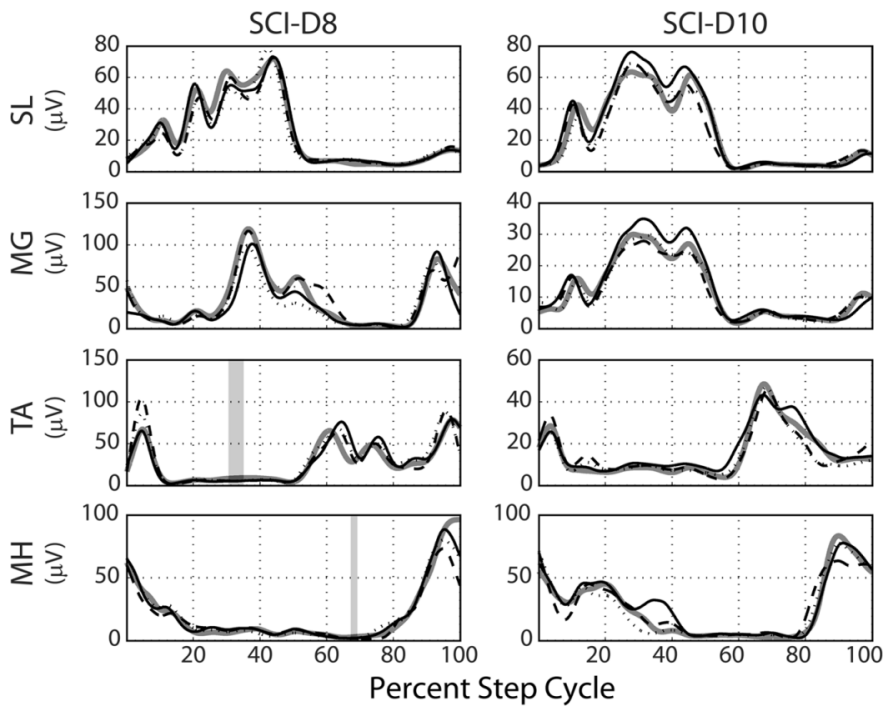

Fig. 5 Effect of trainer assistance alteration on muscle activation for two subjects. Thick solid line is the mean EMG with robot attached yet passive and the trainer assisting as needed for Experiment D. Data from Experiments C and E are not shown, as the representative data shown in Experiment D (robot passive) did not change significantly across runs. Thin solid line is exp. C: robot and trainer both fully active; dotted line is exp. D, robot active and trainer not assisting at the ankle; and dashed line is exp. E: robot active and trainer assisting at the patella only during stance. Shaded areas represent regions of consistently no overlap of $95 \%$ confidence intervals. Abbreviations: soleus (SL), medial gastrocnemius (MG), tibialis anterior (TA), medial hamstrings $(\mathrm{MH})$.

assistance, and $27 \pm 16 \%$ for the patella assistance only condition. When the confidence intervals did not overlap, the mean absolute percent difference in EMG amplitude across subjects and muscles was $5.4 \pm 5.2 \%$, relative to the peak EMG for each muscle during the step cycle.

Analysis of the differences in mean burst amplitude across conditions demonstrated that 20/36 and 16/36 of the comparisons were not significantly different in comparisons 2 and 3, respectively. When the magnitudes were significantly different, the percent differences both increased and decreased with a majority of the differences seen in the SL, TA, and MH and predominantly in subjects SCI-D8 and SCI-D7. The effect of assisting only as needed at the patellar tendon had more measurable effects, significantly decreasing the mean burst magnitude in 15 of the 25 comparisons that could be made. The remaining 11 comparisons could not be made due to noise or absence of activity, phenomenon which were mostly seen in three of the subjects: SCI-C8, SCI-B4, and SCI-D9 and affected all muscles but the soleus (SL).

3) Force as Function of Trainer Assistance Style: The mean force applied by the robot varied between the three assistance conditions. The mean magnitude of force supplied by the robot was calculated for the last ten steps for each of the active assist portions of experiments $\mathrm{C}$-E during swing and averaged across subject and step (Fig. 6). The mean force generated by the robot in order to maintain the stepping pattern was smallest when the trainer assisted only at the knee and not at the ankle $(\mathrm{p}<0.01$, ANOVA, Fig. 6). Thus, the robot guided the subject's shank with the least applied force when the trainer assisted only at the knee, and applied the most force when it was required to drive the shank without popliteal assistance from the trainer.

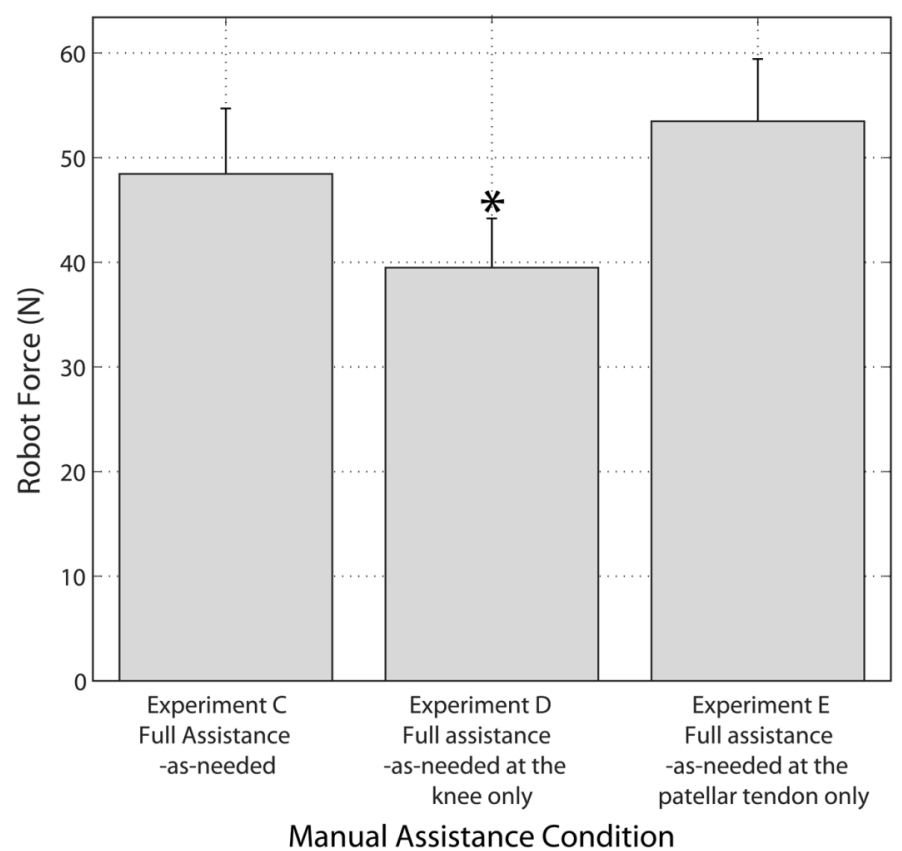

Fig. 6 Mean resultant force for the last ten steps of active assistance for Experiments C-E during swing. Standard error bars are shown. Asterisk (*) indicate a significant difference in robotic force, $\mathrm{p}<0.01$, one way ANOVA.

1) Adaptive Impedance Shaping With an Error Based Update Law: During the adaptive gain experiments, the algorithm (2) shaped the impedance of the robot controller on a step-bystep basis. For reference, the starting impedance values are represented by the large ellipses at the beginning of stance in all of the subfigures [Fig. 7(A)], and the final values are shown at selected locations along the trajectory. Both proportional and derivative gains decreased and reached a steady state after approximately 30 steps [Fig. 7(B)]. The gains converged to similar values for each subject on the two consecutive, identical, experimental runs with the gains initialized to the same large values. Each subject's stepping trajectory was well maintained as the gains adapted: the mean resultant position error during swing in the last ten of the 50 steps in these experiments was $2.0 \pm 0.58$ $\mathrm{cm}$.

Each subject had a unique final set of proportional and derivative gains following convergence, which varied with the phase of the step cycle ([Fig. 7(A)]. For example, subject SCI-C8 in Fig. 7(A) required a stiffer controller in mid-swing through foot placement at heel strike, as evidenced by the large stiffness ellipses at these phases of the step cycle. In contrast, subject SCI-D7 required a stiffer controller at the beginning of swing. From these data, it is clear that the robot was initially very stiff, but that the adaptive gain algorithm greatly reduced and shaped the gains for each subject.

The gains for subject SCI-D7, however, reached a first steady state, and then diverged to a second steady state, resulting in increased proportional gains near the beginning of swing [Fig. 7(B), Px, Py]. The second increase was caused by a medial shift in the foot placement at heel strike, which the robot interpreted as a decreased stepping height, since the medial-lateral motion of the robot was not instrumented and this motion was controlled by the human trainer. Thus, although 
A
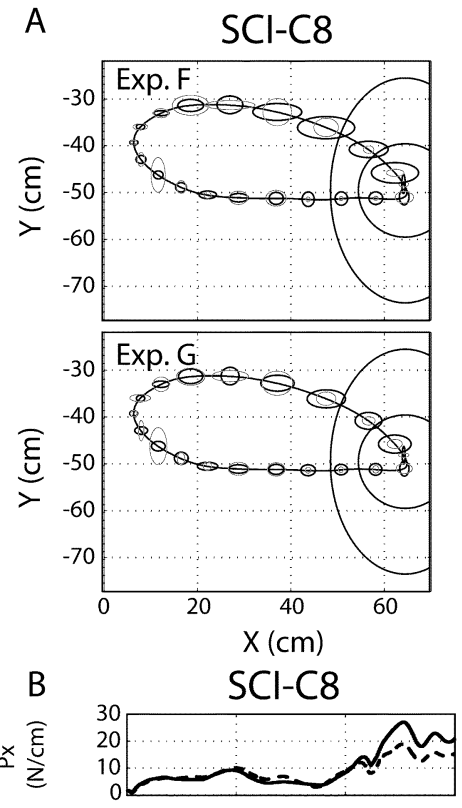

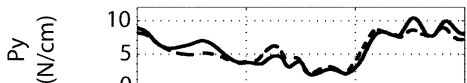

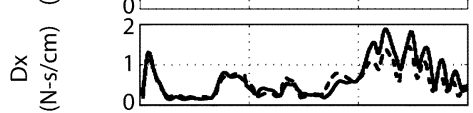

ธิ $\frac{\hat{\Xi}}{\hat{n}}$

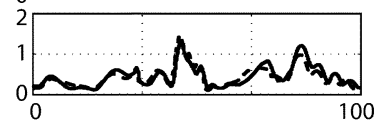

Percent of Step Cycle

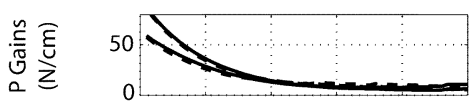

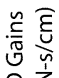

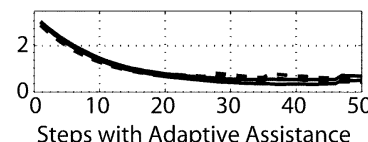

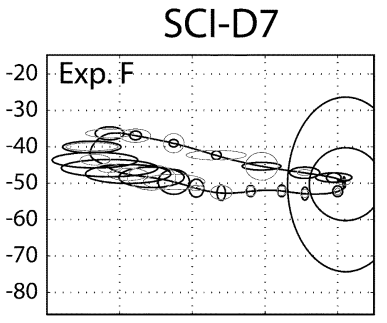

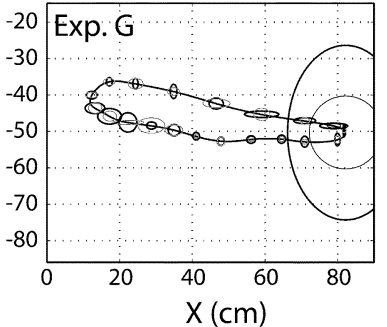

SCl-D7
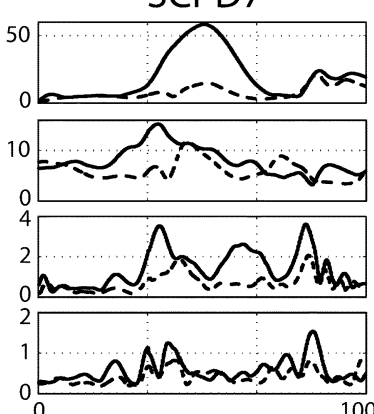

Percent of Step Cycle
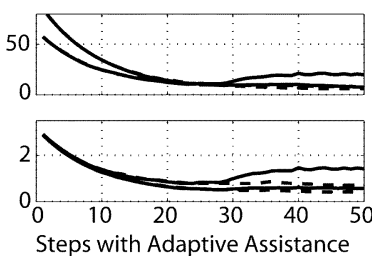

Fig. 7 Adaptive gains for two representative subjects SCI-C8 and SCI-D7. A: Proportional (thick) and derivative (thin) ellipses plotted on the desired trajectory for Experiment F (top) and Experiment G (bottom). Major and minor diameter values are the mean values of the gains for the last ten steps in the field. The large, partially displayed ellipses show the size of the starting stiffness (70 $\mathrm{N} / \mathrm{cm}$ by $105 \mathrm{~N} / \mathrm{cm})$ and damping $(3.5 \mathrm{~N}-\mathrm{s} / \mathrm{cm}$ by $3.5 \mathrm{~N}-\mathrm{s} / \mathrm{cm})$ values. B-Top: Steady state $\mathrm{P}$ and $\mathrm{D}$ gains for the $v$ any $y$ directions. Values are again the mean of the last ten steps. Solid (-) and dashed (-) lines represent data from experiment F and G, respectively. B-Bottom: Convergence of PD gains over 50 steps for both directions and experiments overlaid.

this increase in impedance was not due to an actual change in the subject's stepping ability, it does illustrate the ability of the adaptive algorithm (2) to react to perceived stepping errors. This medial-lateral foot placement change only occurred for one subject.

The reduction of the robot PD gains caused significant increases in the variability of step length and peak step height, and in mean position error during swing for the last ten steps in the adaptive field (Fig. 8, p $<0.05$, one-way ANOVA, comparing values from the last ten steps of Experiment $E$ with static assistance to last ten steps of Experiments $F$ and $G$ with adaptive assistance). Analysis of the differences in mean burst amplitude comparing a stiff to a compliant controller demonstrated that $11 / 30$ were not significantly different (6/30 were significantly
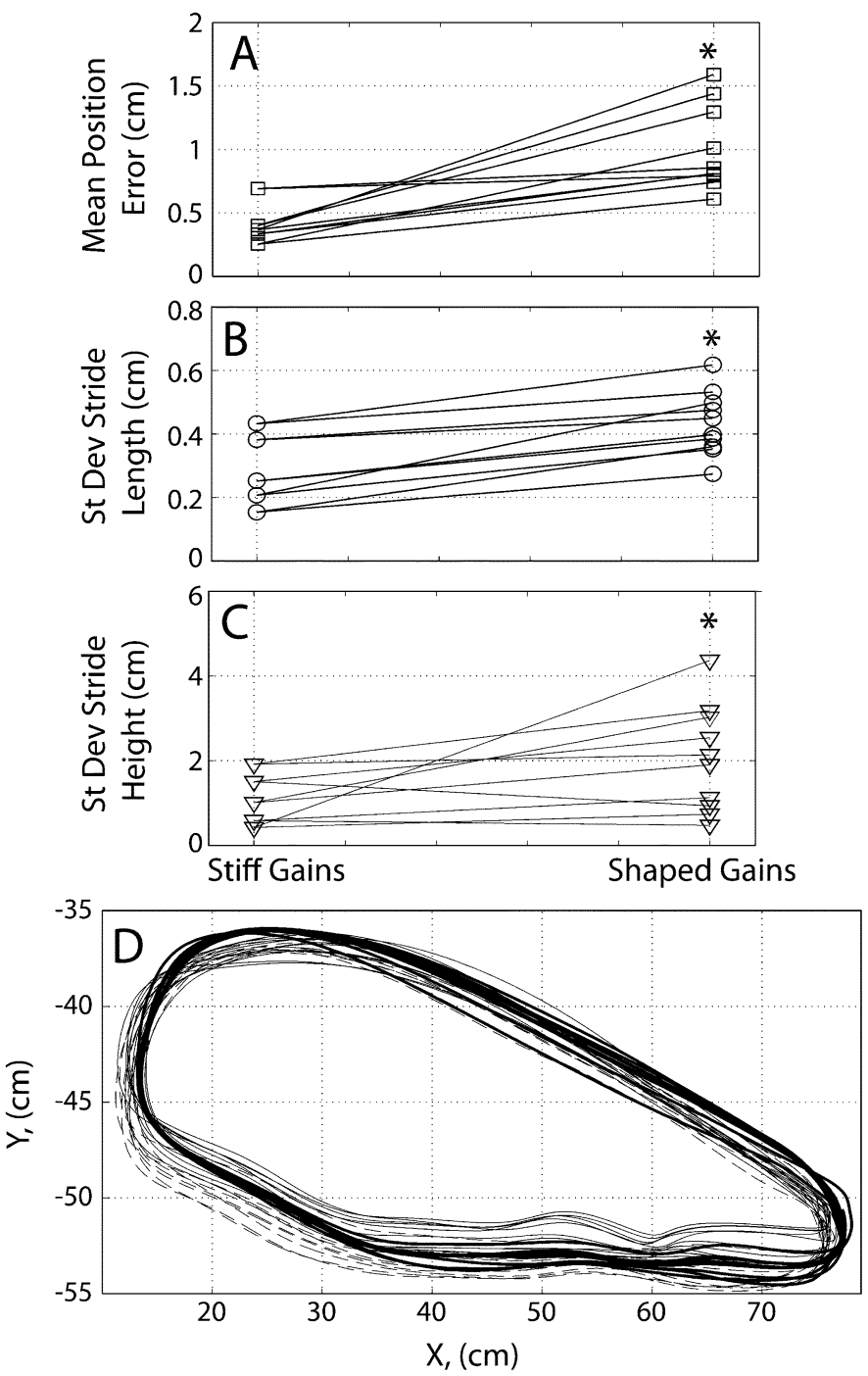

Fig. 8 Effect of Adaptive Algorithm on Force, Error during swing, and Step Variability for Experiments F and G. Condition 1: Last ten steps with patella only trainer assistance (Exp. E) and full robot assistance (stiff gains). Condition 2: Last ten steps of Experiments. F and $\mathrm{G}$ with patella only trainer assistance following 40 steps of shaping of PD gains to subject performance (shaped gains). Shown are A: mean error measured during swing, as well as the B: standard deviation of step length, and C: standard deviation of peak step height. D: Representative data from subject SCI-D9 showing significantly increased variability in step length, step height, and positional errors. The solid line is the position trace during condition 1 and the dashed and solid grey lines are from Condition 2. Asterisk (*) indicates a significant difference, $\mathrm{p}<0.05$, one way ANOVA.

lower). As was the case for other experiments, $13 / 30$ of the comparisons could not be made due to noise or absence of activity. Subject SCI-D10 did not participate in this experiment.

\section{DISCUSSION}

This study demonstrates the feasibility of a manual teach-and-replay procedure for robotic gait training. A trainer manually dictated a desired stepping trajectory on a subject-specific basis by moving the subject's leg with a lightweight robot attached to it. The robot then replayed the trajectory, thereby generating stepping kinematics and muscle activity that were similar to those observed during manually assisted stepping. The key technological innovation was that the robot was back-drivable enough to measure a trainer-assisted 
stepping pattern, and powerful enough to replicate that stepping pattern with the trainer only assisting in knee extension during stance. Second, the adaptive impedance-shaping algorithm that measured tracking errors and included a forgetting process could systematically adjust the impedance of the robot on a step-by-step basis. Using this algorithm, the subjects stepped in a more compliant environment, allowing more step-to-step variability while still maintaining the overall stepping pattern. Impedances evolved in a subject-specific fashion for different phases of the step cycle, in a repeatable way.

In the present study, three different trainers were recruited to assist the right leg of the subjects through stepping trajectories. Three trainers were used because of practical issues with scheduling. All three of these trainers had worked together for several years and were highly skilled in providing manual assistance. It is known, however, that even experienced trainers provide different patterns of assistance for the same individual [23]. The goal in this paper was to study the feasibility of a robotic tool and algorithm that could record a given trainer's induced trajectory and replay it. Future work will include study how the trajectories induced by specific trainers influence gait training outcomes.

We compared the gait pattern induced by the human trainers to the robotic device because the stated potential advantage of robotic devices has consistently been that they will be more cost effective and provide longer treatments than manual assistance. We found EMG activity from skilled trainers providing manual assistance and the robot-induced gait patterns to be similar. It is, however, possible that the EMG activity elicited during assisted walking is not optimal for motor retraining. This of course is a larger research question, which robotic devices such as the one described here may help to answer by virtue of providing a means to apply specific patterns of assistance during training. For the present study, we note that all of the trainers were highly experienced in manual gait training, and that training by this group has produced locomotor patterns in previous studies that are consistent with nondisabled patterns [36].

We also assumed for this study that naturalistic trajectories, individualized to the subject, will be better for training than standardized trajectories. Again, this assumption is unproven. The robotic device described here can provide a means to test this assumption. Similarly, we hypothesized that a gait pattern with more variability is better for training than one with less variability and developed an adaptive impedance control algorithm that selectively reduces impedance during training, allowing more stepping variability. Here, we test the feasibility of this algorithm to increase stepping variability and make no claim regarding the effect on the EMG activity quality after such an increase. Increased variability in a robotic training pattern was recently suggested to improve gait relearning in spinal-transected mice [37], but again this hypothesis is yet to be verified for humans with SCI.

A shortcoming of this experiment is that we did not measure the kinematics of manual assistance without the robot attached. Such a measurement would have allowed determination of whether the trainers provided the same manual assistance regardless of robot attachment. We have found previously that attachment of the robot has little effect on the walking patterns of healthy adult subjects [18]. In addition, the trainers felt that they could generate the desired stepping pattern with the robot attached. However, this issue should still be addressed more carefully in future research.

\section{1) Feasibility of Manual Teach-and-Replay for Robotic} Gait Training: The first versions of robotic gait training devices have relied on prerecorded [13] or mechanically fixed [15] stepping patterns because the devices have relatively high mechanical impedance or limited degrees-of-freedom, thus prohibiting an external source such as a trainer from manually prescribing their motions. The Lokomat research group has recently addressed this issue by developing an algorithm that distorts and time-scales the stepping trajectory based on measurements of the contact force between the robot and the patient [21], [26]. This approach assumes that the contact force reflects the patient's intentional desire for a different trajectory, and thus may have limited applicability for more severely impaired subjects who have greater difficulty producing appropriate kinematics or for patients who have excessive clonus or spasticity [26].

In this paper, trainers provided manual assistance during stepping while a highly back-drivable robot measured and recorded the elicited patterns of stepping. The same robot then replayed a representative mean of the trajectories. We found only minor differences when we compared the muscle activity elicited by the trainer during the "teach" phase, and the muscle activity elicited by the trainer and robot together during the "replay" phase. Thus, this study demonstrates that a step training robot that assists along subject-specific stepping trajectories can elicit locomotor activity that is comparable to that elicited by skilled trainers providing manual assistance. This result is most compelling for the robotic replay condition in which the trainer assisted only at the patella tendon, preventing knee flexion when necessary during stance. In this condition, the robot was solely responsible for assisting the leg through the swing motion, and evoked swing activity comparable to that when a trainer alone assisted swing. The trainer only needed to prevent buckling of the knee during stance, which did not require large forces from her.

Ideally, the act of measuring the trainer-elicited stepping pattern would not alter that pattern. However, we did find some differences in the muscle activity elicited when the trainer stepped the patient without the robot, compared to when the trainer stepped the patient with the robot passively attached (i.e., in "teach mode"). One possible explanation is that the trainer stepped the patient along a different kinematic path when the robot was attached because the trainer had to share the workspace with the robot (i.e., the movement of ARTHuR's links constrained the comfortable arm motions for the trainer). However, we were not able to verify this possibility because we did not measure the stepping kinematics when the robot was not attached. Another possibility is that the differences arose because the robot constrained the degrees of freedom of the leg, limiting medial-lateral hip rotation and ankle inversion/eversion and supination/pronation. A third possibility is that the added inertia of the robot affected the pattern of stepping and thus muscle activity. Engineering optimization of the robot design could reduce each of these factors. Regardless of the reason for 
the moderate differences, the overall pattern of muscle activity was similar with and without the robot attached (Figs. 3 and 4).

2) Adaptive Impedance Shaping: A concern with robotic training is that if the robot assists in a rigid fashion along a fixed trajectory, then the patient's motivation and ability to actively participate in training will be reduced [26]. Rigid assistance may drive the spinal cord into a state of "learned helplessness" [38]-[40] where the nervous system, not challenged to explore potential stepping patterns on its own, will defer to the trainer and cease learning. On the other hand, a robot that allows some amount of errors or variability in stepping could presumably encourage participation and motor learning. In this paper, we used a feedback law with a forgetting factor to adapt a robot's impedance as a function of stepping error. As a result, the impedance decreased from a large initial value with repeated stepping, but only in the parts of the step trajectory where a firm level of assistance was not needed. The pattern of impedance converged repeatedly on separate trials, indicating that the detailed steady-state pattern of impedance generated by the algorithm was not random, but causally associated with the subject's stepping ability. As a result of the selectively decreased impedance, trajectory variability significantly increased, and less force was required from the robot to assist in swing.

Increased variability in robotic assistance has been suggested to speed the recovery of the injured spinal cord relative to low variability training in mice [37], [40]. Cai et al. stepped-trained spinal-transected mice with three training paradigms: a fixed trajectory with low compliance proportional-integral-derivitive (PID) controller; a variable trajectory without inter limb coordination; and a variable trajectory with interlimb coordination. Their results suggest that six weeks of variable training may increase the number of steps completed during a 15-s period as well as increased the rhythmicity and duration of stepping bouts.

Real-time adjustment of the impedance of the robot during robotic step training has been demonstrated previously for the Lokomat [26]. The algorithm proposed here differs in two main ways. First, it reduces the impedance based on a measurement of ongoing kinematic error relative to the desired stepping trajectory, using a learning law modeled after human motor adaptation. The goal is to reduce the robot's assistance and thus allow greater stepping variability if the kinematics of the patient's stepping pattern is near the trainer induced trajectory. In contrast, the Lokomat algorithm decreases the impedance proportionally to the contact force against the robot. The goal in this case is to allow the patient greater freedom in their movements when they wish to change the desired stepping trajectory, or when they are exhibiting a large effort. A question with this approach is how to know when the patient is doing changes that are meaningful and when this not the case (due to spasticity or fatigue for example). The impedance control algorithm developed here is different in that it responds to small errors, not large contact forces, and thus only allows freedom when stepping is consistently along the desired trajectory. In the case of a sudden, unexpected change in stepping pattern due to spasticity, we implemented a second layer of safety in the form of maximum state deviation limits, which would return the device to a passive state if exceeded. Second, the algorithm proposed here adapts the impedance as a function of position throughout the step cycle, while the Lokomat algorithm fixes the gains for the entire step. The result of the algorithm proposed here is that the assistance is shaped to the needs of the patient at each phase of the step cycle.

A key goal of future clinical studies will be to rigorously test the hypothesis that allowing the patient to step along a naturalistic stepping trajectory produces better clinical outcomes than stepping along a standardized trajectory. Similarly, future clinical studies should test the hypothesis that a robot that selectively shapes its impedance based on patient stepping ability, allowing more stepping variability, is more effective than one that does not. The robotic tools and techniques described in this paper provide a way to rigorously test these hypotheses.

\section{ACKNOWLEDGMENT}

The authors would like to thank A. Budovitch and R. van den Brand of the UCLA Human Locomotion Research Center for their assistance in planning and running the experiments, to D. Aoyagi for his assistance in running the experiments, to C. Angeli for her assistance in data analysis, and to R. Edgerton for suggestions on the study design and reviewing the manuscript.

\section{REFERENCES}

[1] H. Barbeau, M. Wainberg, and L. Finch, "Description and application of a system for locomotor rehabilitation," Med. Biol. Eng. Comput., vol. 25, pp. 341-344, 1987.

[2] M. Visintin, H. Barbeau, N. Korner-Bitensky, and N. Mayo, "A new approach to retrain gait in stroke patients through body weight support and treadmill stimulation," Stroke, vol. 29, pp. 1122-8, 1998.

[3] S. Hesse, B. Helm, J. Krajnik, M. Gregoric, and K. H. Mauritz, "Treadmill training with partial body weight support: Influence of body weight release on gait of hemiparetic patients," J. Neuro. Rehab., vol. 11, pp. $15-20,1997$.

[4] A. Wernig, A. Nanassy, and S. Muller, "Laufband (treadmill) therapy in incomplete paraplegia and tetraplegia," J. Neurotrauma, vol. 16, pp. 719-726, 1999.

[5] V. Dietz, G. Colombo, L. Jensen, and L. Baumgartner, "Locomotor capacity of spinal cord in paraplegic patients," Ann. Neurol., vol. 37, pp. 574-582, 1995.

[6] S. J. Harkema, S. L. Hurley, U. K. Patel, P. S. Requejo, B. H. Dobkin, and V. R. Edgerton, "Human lumbosacral spinal cord interprets loading during stepping," J. Neurophysiol., vol. 77, pp. 797-811, 1997.

[7] S. J. Harkema, "Neural plasticity after human spinal cord injury: Application of locomotor training to the rehabilitation of walking," Neuroscientist, vol. 7, pp. 455-468, 2001.

[8] V. Dietz, M. Wirz, G. Colombo, and A. Curt, "Locomotor capacity and recovery of spinal cord function in paraplegic patients: A clinical and electrophysiological evaluation," Electroenceph. Clin. Neurophys., vol. 109, pp. 140-153, 1998.

[9] V. Dietz, R. Mueller, and G. Colombo, "Locomotor activity in spinal man: Significance of afferent input from joint and load receptors," Brain, vol. 125, pp. 2626-2634, 2002.

[10] V. R. Edgerton, R. R. Roy, J. A. Hodgson, R. J. Gregor, and C. P. de Guzman, "Recovery of full weight-supporting locomotion of the hindlimbs after complete thoracic spinalization of adult and neonatal cats," in Restorative Neurology, Plasticity of Motoneuronal Connections. New York: Elsevier, 1991, pp. 405-418.

[11] V. R. Edgerton, R. D. de Leon, N. Tillakaratne, M. R. Recktenwald, J. A. Hodgson, and R. R. Roy, "Use-dependent plasticity in spinal stepping and standing," in Advances in Neurology: Neuronal Regeneration, Reorganization and Repair. Philadelphia, PA: LippincottRaven, 1997, pp. 233-247.

[12] V. R. Edgerton, R. D. de Leon, S. J. Harkema, J. A. Hodgson, N. London, D. J. Reinkensmeyer, R. R. Roy, R. J. Talmadge, N. J. Tillakaratne, W. Timoszyk, and A. Tobin, "Retraining the injured spinal cord.," J. Physiol., vol. 533, pp. 15-22, 2001.

[13] G. Colombo, M. Joerg, R. Schreier, and V. Dietz, "Treadmill training of paraplegic patients with a robotic orthosis," J. Rehab. Res. Devel., vol. 37, pp. 693-700, 2000. 
[14] G. Colombo, M. Wirz, and V. Dietz, "Driven gait orthosis for improvement of locomotor training in paraplegic patients," Spinal Cord, vol. 39, pp. $252-5,2001$.

[15] S. Hesse, C. Werner, D. Uhlenbrock, S. von Frankenberg, A. Bardeleben, and B. Brandl-Hesse, "An electromechanical gait trainer for restoration of gait in hemiparetic stroke patients: Preliminary results," Neurorehab. Neural Repair, vol. 15, pp. 37-48, 2001.

[16] S. Hesse and C. Werner, "Partial body weight supported treadmill training for gait recovery following stroke," Adv. Neurol., vol. 92, pp. 423-428, 2003.

[17] C. Werner, S. Von Frankenberg, T. Treig, M. Konrad, and S. Hesse, "Treadmill training with partial body weight support and an electromechanical gait trainer for restoration of gait in subacute stroke patients: A randomized crossover study," Stroke, vol. 33, pp. 2895-2901, 2002.

[18] J. L. Emken, J. H. Wynne, S. J. Harkema, and D. J. Reinkensmeyer, "A robotic device for manipulating human stepping," IEEE Trans. Robotics, vol. 22, no. 1, pp. 185-189, Feb. 2006.

[19] M. Wirz, D. H. Zemon, R. Rupp, A. Scheel, G. Colombo, V. Dietz, and T. G. Hornby, "Effectiveness of automated locomotor training in patients with chronic incomplete spinal cord injury: A multicenter trial," Arch. Phys. Med. Rehabil., vol. 86, pp. 672-680, 2005.

[20] V. Dietz, M. Wirz, A. Curt, and G. Colombo, "Locomotor pattern in paraplegic patients: Training effects and recovery of spinal cord function," Spinal Cord, vol. 36, pp. 380-90, 1998.

[21] S. Jezernik, G. Colombo, and M. Morari, "Automatic gait-pattern adaptation algorithms for rehabilitation with a 4-DOF robotic orthosis," IEEE Trans. Robotics Autom., vol. 20, no. 3, pp. 574-582, Jun. 2004.

[22] J. M. Hilder and A. E. Wall, "Alterations in muscle activation patterns during robotic-assisted walking," Clin. Biomech., vol. 20, pp. 184-193, 2005.

[23] J. A. Galvez, G. Kerdanyan, S. Maneekobkunwong, R. Weber, M. Scott, S. J. Harkema, and D. J. Reinkensmeyer, "Measuring human trainers' skill for the design of better robot control algorithms for gait training after spinal cord injury," in Proc. IEEE 9th Int. Conf. Rehab. Robotics, Jun. 28-Jul. 1, 2005, pp. 231-234.

[24] S. Hesse, D. Uhlenbrock, C. Werner, and A. Bardeleben, "A mechanized gait trainer for restoring gait in nonambulatory subjects," Arch. Phys. Med. Rehab., vol. 81, pp. 1158-1161, 2000.

[25] H. Schmidt, D. Sorowka, S. Hesse, and R. Bernhardt, "Robotic walking simulator for neurological gait rehabilitation," in Proc. 2nd Joint EMBS/BMES Conf., Oct. 23-26, 2002, vol. 3, pp. 2356-2357.

[26] R. Riener, L. Lunenburger, S. Jezernik, M. Anderschitz, G. Colombo, and V. Dietz, "Patient-cooperative strategies for robot-aided treadmill training: First experimental results," IEEE Trans. Neural Syst. Rehab. Eng., vol. 13, no. 3, pp. 380-394, Sep. 2005.

[27] D. J. Reinkensmeyer, D. Aoyagi, J. L. Emken, J. Galvez, W. E. Ichinose, G. Kerdanyan, J. A. Nessler, S. Maneekobkunwong, W. Timoszyk, K. Vallance, R. Weber, J. Wynne, R. D. de Leon, J. E. Bobrow, S. Harkema, and V. R. Edgerton, "Robotic gait training: Toward more natural movements and optimal training algorithms," in Proc. 26th Annu. EMBC, 2004, pp. 4818-4821.

[28] J. L. Emken, J. E. Bobrow, and D. J. Reinkensmeyer, "Robotic movement training as an optimization problem: Designing a controller that assists only as needed," in Proc. 9th Int. Conf. Rehab. Robotics. (ICORR'05), Jun. 28-Jul. 1, 2005, pp. 307-312.

[29] D. Aoyagi, W. E. Ichinose, S. Harkema, D. J. Reinkensmeyer, and J. E. Bobrow, "An assistance robotic device that can synchronize to the pelvic motion during human gait training," in Proc. 9th Int. Conf. Rehab. Robotics. (ICORR'05), Jun. 28-Jul. 1, 2005, pp. 565-568.

[30] F. M. Maynard, M. B. Bracken, G. Creasey, J. F. Ditunno, W. H. Donovan, T. B. Ducker, S. L. Garber, R. J. Marino, S. L. Stover, C. H. Tator, R. L. Waters, J. E. Wilberger, and W. Young, "International standards for neurological and functional classification of spinal cord injury," Spinal Cord, pp. 266-274, 1997.

[31] J. L. Emken and D. J. Reinkensmeyer, "Robot-enhanced motor learning: Accelerating internal model formation during locomotion by transient dynamic amplification," IEEE Trans. Neural Syst. Rehab. Eng., vol. 13, no. 1, pp. 33-39, Mar. 2005.

[32] D. J. Reinkensmeyer, J. L. Emken, J. Liu, and J. E. Bobrow, "The nervous system appears to minimize a weighted sum of kinematic error, force, and change in force when adapting to viscous environments during reaching and stepping," presented at the Adv. Comput. Motor Control III, San Diego, CA, 2004 [Online]. Available: http://www.bme.jhu.edu/acmc/, unpublished

[33] D. J. Reinkensmeyer, J. Liu, and J. L. Emken, "Robotic enhancement of human motor learning based on computational modeling of neural adaptation," presented at the ASME IMECE, Anaheim, CA, 2004, unpublished.
[34] D. J. Reinkensmeyer, D. Aoyagi, J. L. Emken, J. Galvez, W. E. Ichinose, G. Kerdanyan, S. Maneekobkunwong, J. A. Minakata, J. A. Nessler, R. Weber, R. R. Roy, R. de Leon, J. E. Bobrow, S. J. Harkema, and V. R. Edgerton, "Tools for understanding and optimizing robotic gait training," J. Rehab. Res. Devel., vol. 43, no. 5, pp. 657-670, Aug.-Sep. 2006.

[35] J. A. Beres-Jones and H. S. J. , "The human spinal cord interprets velocity-dependent afferent input during stepping.," Brain, vol. 127, pp. 2232-2246, 2004.

[36] V. Dietz and S. J. Harkema, "Locomotor activity in spinal cord-injured persons," J. Appl. Physiol., vol. 95, pp. 1954-1960, 2004.

[37] L. L. Cai, A. J. Fong, C. K. Otoshi, Y. Liang, J. W. Burdick, R. R. Roy, and V. R. Edgerton, "Implications of assist-as-needed robotic step training after a complete spinal cord injury on intrinsic strategies of motor learning.," J. Neurosci., vol. 26, pp. 10564-10568, 2006.

[38] R. N. Wool, D. Siegel, and P. R. Fine, "Task performance in spinal cord injury: Effects of helplessness training," Arch. Phys. Med. Rehab., vol. 61, pp. 321-325, 1980.

[39] J. W. Grau, D. G. Barstow, and R. L. Joynes, "Instrumental learning within the spinal cord: I. Behavioral properties," Behav. Neurosci., vol. 112, pp. 1366-1386, 1998

[40] L. L. Cai, A. J. Fong, C. K. Otoshi, Y. Q. Liang, J. G. Cham, H. Zhong, R. R. Roy, V. R. Edgerton, and J. W. Burdick, "Effects of consistency vs. variability in robotically controlled training of stepping in adult spinal mice," in Proc. IEEE 9th Int. Conf. Rehab. Robotics (ICORR'05), Jun. 28-Jul. 1, 2005, pp. 575-579.

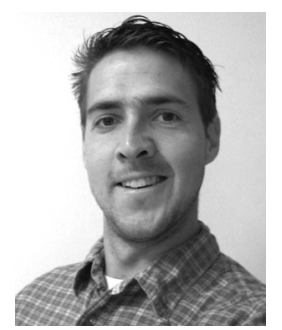

Jeremy L. Emken received the B.Sc. degree in mechanical engineering from California Polytechnic State University, San Luis Obispo, CA, in 1998 and the M.Sc. and Ph.D. degrees in biomedical engineering from the University of California-Irvine, Irvine, CA, in 2003, and 2006, respectively.

$\mathrm{He}$ was a Research Engineer with Medtronic Vascular, Santa Rosa, CA, from 1999 to 2001 . He is currently a Research Associate at the California Institute of Technology, Pasadena, developing a novel neural prosthetic device. His research interests include brain-computer interfaces, neural prosthetics, neuroscience, motor learning, and robot-assisted rehabilitation.

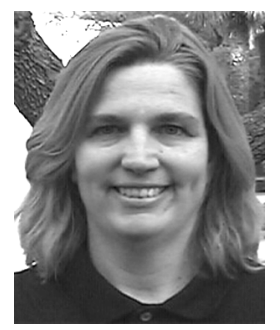

Susan J. Harkema received the B.S. and Ph.D. degrees in physiology from Michigan State University, East Lansing.

She held a Postdoctoral Fellowship in neurology at the University of California, Los Angeles. She was an Assistant Professor in the Department of Neurology and the Brain Research Institute. Currently, she is an Associate Professor in the University of Louisville School of Medicine's Department of Neurological Surgery, Louisville, KY. She serves as a Rehabilitation Director of the Kentucky Spinal Cord Injury Research Center and Director of Research at the Frazier Rehab Institute. The focus of her research is to study neural mechanisms for human locomotion and the level of plasticity after neurologic injury.

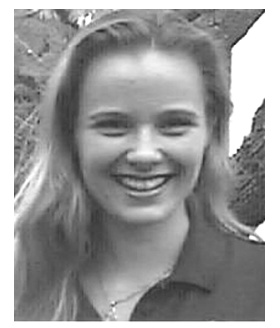

Janell A. Beres-Jones received the B.S. and M.S. degrees in physiological science from the University of California, Los Angeles (UCLA), in 1997 and 2000, respectively.

She spent eight years working as a Research Assistant and Research Supervisor at the Human Locomotion Research Center (HLRC), UCLA, investigating sensory processing and plasticity of the human spinal cord with regard to the neural control of locomotion in humans. She is currently a Consultant teaching clinicians how to implement Locomotor Training, a novel rehabilitative technique for the recovery of walking after neurologic injury, which emerged from the research at the HLRC. 


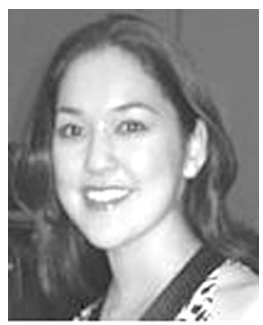

Christie K. Ferreira received the B.S. degree in physiological science from the University of California, Los Angeles (UCLA), in 2002.

She was a Student Research Assistant for two years and a Research Associate Supervisor at UCLA with Dr. Harkema for four years. She continues her research with Dr. Harkema at the University of Louisville, Louisville, KY, and the Frazier Rehab Institute, Louisville. Her research interests include spinal reflexes, cardiovascular adaptations, and electromyographic patterns after stand and step training in the spinal cord-injured population.

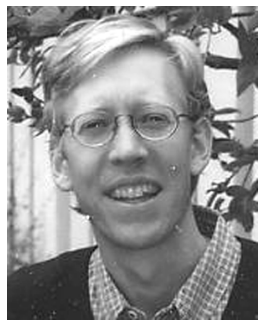

David J. Reinkensmeyer (S'93-M'93) received the B.S. degree in electrical engineering from the Massachusetts Institute of Technology, Cambridge, and the M.S. and Ph.D. degrees in electrical engineering from the University of California, Berkeley, in 1988, 1991, and 1993, respectively.

He was a Research Associate at the Rehabilitation Institute of Chicago and Northwestern University Medical School from 1994 to 1997. He is currently an Associate Professor in the Department of Mechanical and Aerospace Engineering and the Department of Biomedical Engineering at the University of California, Irvine. His research interests are in neuromuscular control, motor learning, robotics, and neurorehabilitation. 